\title{
Biometría de las Conexiones Intertendinosas del Dorso de la Mano humana
}

\author{
Biometry of the Intertendinous Connection of the Dorsum of the Human Hand
}

\author{
Alejandro Bruna ${ }^{1,2}$; Andrés Riveros ${ }^{3,4}$ \& Enrique Olave ${ }^{5}$
}

BRUNA, A.; RIVEROS, A. \& OLAVE, E. Biometría de las conexiones intertendinosas del dorso de la mano humana. Int. J. Morphol., 38(5):1165-1172, 2020.

RESUMEN: Las conexiones intertendinosas (CIT) son bandas estrechas de tejido conectivo que unen tendones adyacentes del músculo extensor de los dedos. Dichas bandas se ubican de manera superficial en el dorso del segundo, tercer y cuarto espacios intermetacarpianos (EIMC) presentando funciones importantes como colaborar en la estabilización de la articulación metacarpofalángica y permitir la redistribución de fuerzas extensoras sobre esta articulación. El objetivo del presente estudio fue establecer la biometría de las CIT. Para ello se utilizaron 24 miembros superiores formolizados, pertenecientes al laboratorio de Anatomía, de la Universidad Andrés Bello, sede Viña del Mar, Chile. Se registraron las longitudes de inserción lateral y medial, el ancho y la longitud total de cada CIT. Siguiendo dos criterios, las CIT se clasificaron según morfología y además se localizaron según tercios del dorso de la mano. De las 69 CIT encontradas, el $39 \%$ presentó una disposición transversa y 61 \% una oblicua. En relación a la clasificación morfológica de la CIT se identificó un $29 \%$ del tipo 1; $39 \%$ del tipo 2. Para el caso del tipo 3, un 20 \% presentó la forma "r" y en un $12 \%$ la forma "y". Respecto a su localización se estableció que en el segundo EIMC el $18 \%$ de las CIT se ubicaron el tercio distal del dorso y el $82 \%$ en el tercio medio. En el tercer EIMC el $96 \%$ de las CIT se localizó en el tercio distal y $4 \%$ en el tercio medio. En el cuarto EIMC el $96 \%$ se ubicó en el tercio distal y el $4 \%$ en el tercio medio. Considerando como referencia la línea biestiloidea, las CIT en el segundo EIMC presentaron un promedio de ancho de $8,16 \pm 1,94 \mathrm{~mm}$ y una longitud total promedio de 13,71 \pm 4,70 mm. En el tercer EIMC, un promedio de 10,94 \pm 4,39 $\mathrm{mm}$ para el ancho y 6,29 $\pm 3,26 \mathrm{~mm}$ de longitud total promedio. En el cuarto EIMC el promedio de ancho fue de 7,00 $\pm 3,06 \mathrm{~mm}$ y la longitud total promedio 7,08 \pm 4,42 $\mathrm{mm}$. Estos hallazgos aportan datos respecto de la biometría y localización de las CIT, lo que resulta de utilidad en procedimientos de reparación quirúrgica del dorso de la mano humana.

PALABRAS CLAVE: Anatomía; Conexiones intertendinosas; Juncturae tendinum; Músculo Extensor de los Dedos.

\section{INTRODUCCIÓN}

La realización de las diversas actividades de la mano es la resultante de una delicada interacción de fuerzas flexoras y extensoras que se alternan para desarrollar complejas habilidades que incluyen la prensión o la pinza digital (Chinchalkar et al., 2015). Uno de los músculos más importantes en esta interacción es el músculo extensor de los dedos (ED), el cual se dispone desde el plano superficial del compartimiento posterior del antebrazo para dividirse distalmente en tres fascículos que darán origen a dos tendones dirigidos a la región dorsal de los dedos índice y medio, a lo que se agrega un tendón común destinados a los dedos anular y mínimo. (Llusa et al., 2004; Rouvière \& Delmas, 2005). Asociados a la cuarta corredera del retináculo extensor del carpo, los tendones del músculo ED en la proximidad de la articulación metacarpofalángica $(\mathrm{MCF})$ presentan conexiones intertendinosas (CIT) también conocidas como Juncturae tendinum (Nómina Anatómica de Basilea; His, 1895) y que actualmente conocemos como Connexus intertendinei (Comité Federal sobre Terminología Anatómica, 2001).

Estas CIT corresponden a bandas estrechas de tejido conectivo que unen tendones adyacentes del músculo ED (von Schroeder et al., 1990; Standring, 2016). Dichas bandas que ocupan parte de la región dorsal superficial del segundo, tercer y cuarto espacio intermetacarpiano (EIMC) presentan funciones importantes como colaborar en la esta-

\footnotetext{
${ }^{1}$ Departamento de Morfología, Facultad de Medicina, Universidad Andrés Bello, Viña del Mar, Chile.

${ }^{2}$ Programa de Magíster en Ciencias, Mención Morfología, Facultad de Medicina, Universidad de La Frontera, Temuco, Chile.

${ }^{3}$ Departamento de Ciencias Morfológicas, Facultad de Medicina y Ciencia, Universidad San Sebastián, Lientur 1457, Concepción 4080871, Chile.

${ }^{4}$ Programa de Doctorado en Ciencias Morfológicas, Universidad de La Frontera, Temuco, Chile.

${ }^{5}$ Facultad de Medicina, Universidad de La Frontera, Temuco, Chile.
} 
bilización de la articulación MCF y permitir la redistribución de fuerzas extensoras sobre esta misma (Standring; Kalkisim et al., 2018). Determinantes en limitar la independencia funcional de los dedos, particularmente del dedo anular también evitan la extensión de laceraciones tendinosas, así mismo son tomadas como referencia quirúrgica para la identificación de los diferentes tendones que se disponen en el dorso de la mano (von Schroeder et al.; Pinar et al., 2009). En este sentido, Zancolli (2014), describió que al cerrar el puño de la mano, estas CIT forman un tendón transverso que establecen una fuerza de dirección medial generada por la tracción realizada por el descenso y separación de la cabeza del cuarto y quinto huesos metacarpianos. Lo anterior estaría directamente asociado a que en casos de ausencia o interrupción traumática de estas CIT se puede producir una subluxación del tendón del músculo ED a la altura de la articulación MCF (von Schroeder et al.).

Un aspecto relevante en la descripción de estas CIT tiene relación a su forma. Al respecto von Schroeder et al., propusieron una clasificación morfológica en tres tipos de CIT. A las tipo 1 las describió como delgadas bandas fibrosas de disposición transversa. Para el caso de las CIT tipo 2 señalaron que corresponden a bandas de mayor grosor que frecuentemente presentan una forma romboidal. Respecto a las CIT tipo 3, se diferenciaron dos subtipos, el formato 3 "y" y 3 " $r$ "; en el primero se describe como una división de un tendón del ED en dos ramas, siendo una de ellas una conexión larga y oblicua; en cambio, el segundo formato corresponde a una extensión de un tendón del ED similar a una "rama lateral de un árbol". En ambos casos, el formato "y" y "r" del tipo 3 corresponde a CIT más gruesas que los restantes tipos (Hirai et al., 2001; Celik et al., 2008; Pinar et al.; Palatty et al., 2014; Kalkisim et al.). Bajo un análisis histológico, estas CIT también presentan diferencias. Las tipo 1 se caracterizan por ser un tejido conectivo laxo que presenta combinación de zonas de aspecto fibroso y otras de aspecto tendinoso. Indistinto de la zona, estas fibras se orientan en una sola dirección y siempre paralelas entre sí. En el caso de las CIT tipo 2, se mantuvo la disposición paralela pero su aspecto evidenciaba una mayor densidad de fibras. A diferencia de las anteriores, las CIT tipo 3 presentan fibras organizadas en dos capas dispuestas en diferentes ángulos, uno transverso y otro con fibras oblicuas. Dichas descripción abarca tanto las CIT tipo 3 "y" como también las 3 "r" (Pinar et al.).

Respecto de la ubicación de estos diferentes tipos de CIT, varios estudios reportaron que las CIT que predominan en el segundo EIMC son las tipo 1. En este caso, su prevalencia de presentación varía entre un $57 \%$ y un $87 \%$ de los casos (von Schroeder et al., Pinar et al.; Yammine, 2015). Para el caso del tercer EIMC, el tipo 2 es el que se presenta de forma más frecuente, presentando un intervalo de prevalencia que se extiende entre el $40 \%$ y el $59 \%$ de los casos reportados (Celik et al.; Yammine). En esta misma línea, el tipo de CIT que predomina en el cuarto EIMC es la tipo 3. Al respecto von Schroeder et al. y Palatty et al., señalaron que la mayor prevalencia la presenta el tipo 3 "r", en cambio, Hirai et al., y Pinar et al., informaron una mayor frecuencia de presentación del tipo 3 "y".

En relación a las mediciones biométricas de las CIT, existen reportes de la longitud total y ancho de estas según el EIMC combinado con su tipo de presentación. En el segundo EIMC, donde predominan las CIT tipo 1, su longitud varía entre 6,4 y 8,7 mm y su ancho fluctúa entre 9 y $12 \mathrm{~mm}$. Para el caso de las CIT del tercer EIMC donde mayoritariamente son del tipo 2, presentan entre 8 y $11 \mathrm{~mm}$ de longitud total y entre 5 y $8,7 \mathrm{~mm}$ de ancho. En el cuarto EIMC, donde predominan las CIT tipo 3, la longitud total de las CIT tipo 3 "r" se aproxima a los $12 \mathrm{~mm}$, mientras que las tipo 3 "y" fluctúan entre 10 y $15 \mathrm{~mm}$. Respecto del ancho de estas dos formas, este varía entre 3,8 y 4,3 para la forma 3 " $r$ " y entre 3,6 y 4,2 mm para el caso de la forma 3 "y". (von Schroeder et al.; Celik et al.; Pinar et al.; Palatty et al.).

Otro elemento de interés en la descripción de las CIT tiene relación con variaciones anatómicas que implican la presencia de tendones supernumerarios y/o la ausencia de tendones del músculo ED. Respecto de lo primero, Tountas \& Bergmann (1993), relataron la presencia de un tendón supernumerario hacia el dedo medio de la mano originado desde el músculo extensor del dedo índice. Dicha descripción que alcanza al $10 \%$ de los casos, se suma a reportes respecto de la existencia de un músculo extensor corto de los dedos, tendón doble desde el músculo extensor del dedo índice o músculo extensor propio del dedo medio (Steichen \& Petersen, 1984; von Schroeder \& Botte, 1995). Al respecto Le Double (1897), describió casos en la cual el músculo ED presentó desde 6 hasta 12 tendones, lo que implica que algunos o todos los tendones del ED se presentaron dobles o triples. En esta línea, también existen descripciones de la presencia de dos tendones de inserción por parte del músculo extensor del dedo mínimo (Testut \& Latarjet, 1972; Godwin \& Ellis, 1992).

Si bien son escasos los reportes que describen CIT entre un tendón del ED y los eventuales tendones supernumerarios, von Schroeder \& Botte (1995) reportaron en el $53 \%$ de sus casos, la ausencia del tendón que el músculo ED destina hacia el dedo mínimo. Asimismo, en el $96 \%$ de estos casos, la mencionada ausencia está asociada con la presencia de un tendón doble de este músculo para el dedo anular, a lo que se suma la existencia de un CIT tipo 3 de gran grosor que sustituye al tendón ausente para el dedo mínimo. 
Considerando lo anterior, el objetivo del presente estudio fue describir los aspectos biométricos de las CIT de la mano humana, clasificar cada CIT según su morfología y establecer el predominio en cada EIMC. Adicionalmente, se estableció la ubicación de las CIT según los tercios del dorso de la mano. Finalmente, se describió la presencia de variantes anatómicas tendinosas y la relación de éstas con las CIT. Estos hallazgos toman relevancia por su aporte anátomo-quirúrgico respecto a la caracterización biométrica de los elementos de la región dorsal de la mano humana.

\section{MATERIAL Y MÉTODO}

La presente investigación fue realizada en 24 miembros superiores, (4 derechos, 20 izquierdos), de los cuales el $83 \%$ correspondió a miembros superiores izquierdos. Dicho material cadavérico pertenece a los Laboratorios de Anatomía Humana del Departamento de Morfología de la Facultad de Medicina de la Universidad Andrés Bello, Viña del Mar, Chile. Dichos cadáveres fueron sometidos a un protocolo de fijación con formaldehído al $10 \%$ para su posterior conservación en cámara de frío a $4{ }^{\circ} \mathrm{C}$. Todas las muestras incluidas en el estudio no presentaban signos macroscópicos de disecciones previas, patologías ni cirugías en el dorso de la mano.

Esta investigación se realizó utilizando una metodología de carácter cuantitativo, no experimental, transeccional y descriptiva; para ello, el proceso de disección se inició realizando dos cortes transversos en la piel del dorso de la mano, el primero a la altura de la línea biestiloidea (LBEst) y el segundo a la altura del extremo proximal de la base de la falange proximal de cada uno de los cinco dedos. Posteriormente, estos dos cortes se unieron a través de un un tercer corte longitudinal dispuesto entre el punto medio de los dos cortes transversos mencionados anteriormente. Realizados estos cortes y mediante una disección por planos, se procedió a retirar la piel, el tejido subcutáneo, la fascia superficial y el conjunto de elementos neurovasculares superficiales. Lo anterior permitió exponer las estructuras tendinosas de esta zona y las CIT. Para la realización de esta disección se utilizó material quirúrgico ad hoc y una lupa luminiscente de 4X.

Identificada la CIT se registró la ubicación de cada una de ellas en los diferentes EIMC. Asimismo se establecieron parámetros biométricos como ancho y longitud; para ello y producto de las diversas formas que tuvieron, se requirió medir en primer lugar la longitud medial y lateral de cada una de ellas (Fig. 1). Identificado el punto medio de las longitudes medial y lateral, se pudo establecer el ancho de la CIT. Enseguida y considerando el punto medio del ancho de la CIT, se estableció la longitud de la misma.

Considerando como referencia la LBEst y la articulación MCF de cada dedo se midieron las distancias entre estas referencias y el punto medio de las longitudes medial y lateral de cada CIT (Fig. 1 punto I y II). Complementariamente y tomando las mismas referencias respecto del punto medio de la longitud total, se localizó cada CIT según los tercios proximal, intermedio y distal del dorso de cada mano.

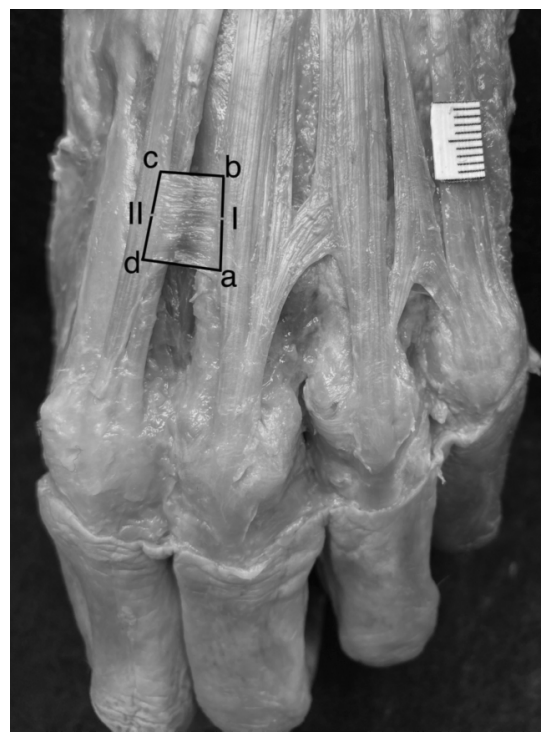

Fig. 1. Vista dorsal mano izquierda. a: punto distal de inserción medial. b: punto proximal de inserción medial. c: punto próxima de inserción lateral. d: punto distal de inserción lateral. I: punto medio de la longitud medial. II: punto medio de la longitud lateral. Longitud medial: distancia entre los puntos a y b. Longitud lateral: distancia entre los puntos c y d. Ancho total: distancia entre los puntos I y II.

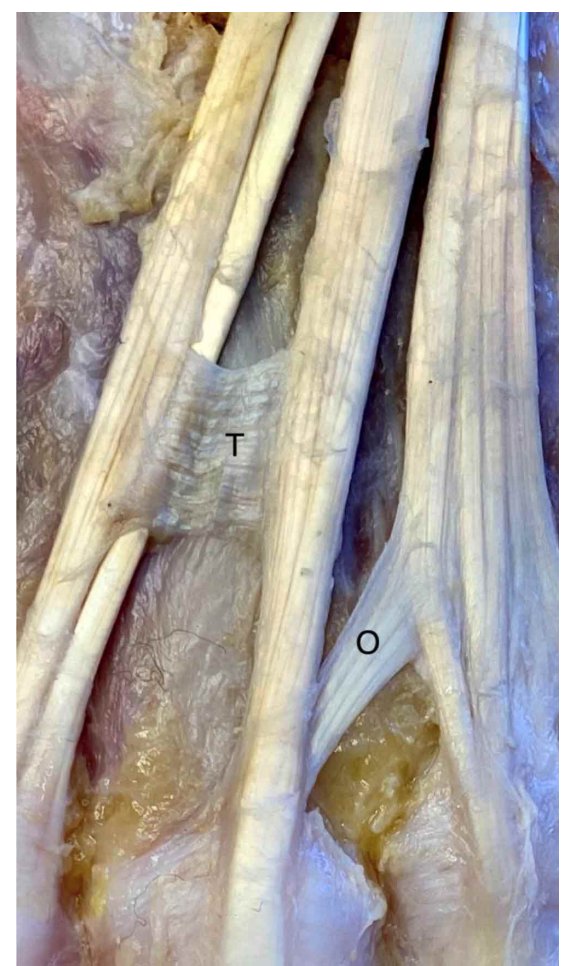

Fig. 2. Vista dorsal mano izquierda.

T: Con ex i ón intertendinosa transversa.

$\mathrm{O}:$ Con ex ión intertendinosa oblicua. 
Adicionalmente se determinó la distribución general según la disposición de sus fibras de las CIT, (transversa u oblicua) junto con establecer cual predominó en cada EIMC. Junto con lo anterior y siguiendo las descripciones de von Schroeder et al. se clasificó cada una de las CIT en tipo 1,2,3 "r" y 3 "y".

Las diferentes mediciones fueron realizadas con un caliper digital Mitutoyo ${ }^{\circledR}$ de 0,01 mm de precisión. En la misma línea, los resultados fueron ingresados a una base de datos Microsoft Excel ${ }^{\circledR}$ y se calcularon las medidas de tendencia central de cada una de las variables registradas. El registro fotográfico de la disección de las muestras fue realizado por una Cámara frontal TrueDepth de 12 MP Apple®.

\section{RESULTADOS}

Respecto a la ubicación de las CIT, éstas no se encontraron en el primer EIMC. En el segundo EIMC, esta conexión estuvo presente en el $92 \%$ de los casos, mientras que en el tercer EIMC fue hallada en el $100 \%$ de la muestra. En el caso del cuarto EIMC este hallazgo alcanzó un $96 \%$ de los casos.

Respecto de la disposición de las fibras, de las 69 CIT encontradas, el $39 \%$ de éstas presentaron una disposición transversa y el $61 \%$ oblicua. Analizado esto por EIMC, en el segundo se encontraron CIT con una disposición transversa en el $91 \%$ y oblicua en el $9 \%$ de las manos. En el tercer EIMC se observaron CIT con disposición transversa en el 12 $\%$ y oblicua en $88 \%$ de los casos. La disposición oblicua fue la más frecuente en el cuarto EIMC con un $79 \%$ versus las CIT con fibras dispuestas en sentido transverso que se presentaron en un $17 \%$ de los casos (Fig. 2).

Siguiendo las clasificación de von Schroeder et al., del total de CIT, se identificaron $29 \%$ del tipo 1 y $39 \%$ del tipo 2. Para el caso del tipo 3, se encontraron $20 \%$ de la forma "r" y $12 \%$ de la forma "y" (Fig. 3A y 3B). Al analizar esta clasificación morfológica según cada EIMC nos encontramos que en el segundo el tipo 1 predominó con un $73 \%$ versus un $27 \%$ de CIT del tipo 2; no encontrando CIT del tipo 3. Para el caso del tercer EIMC, el tipo 1 se presentó en el $8 \%$ de las

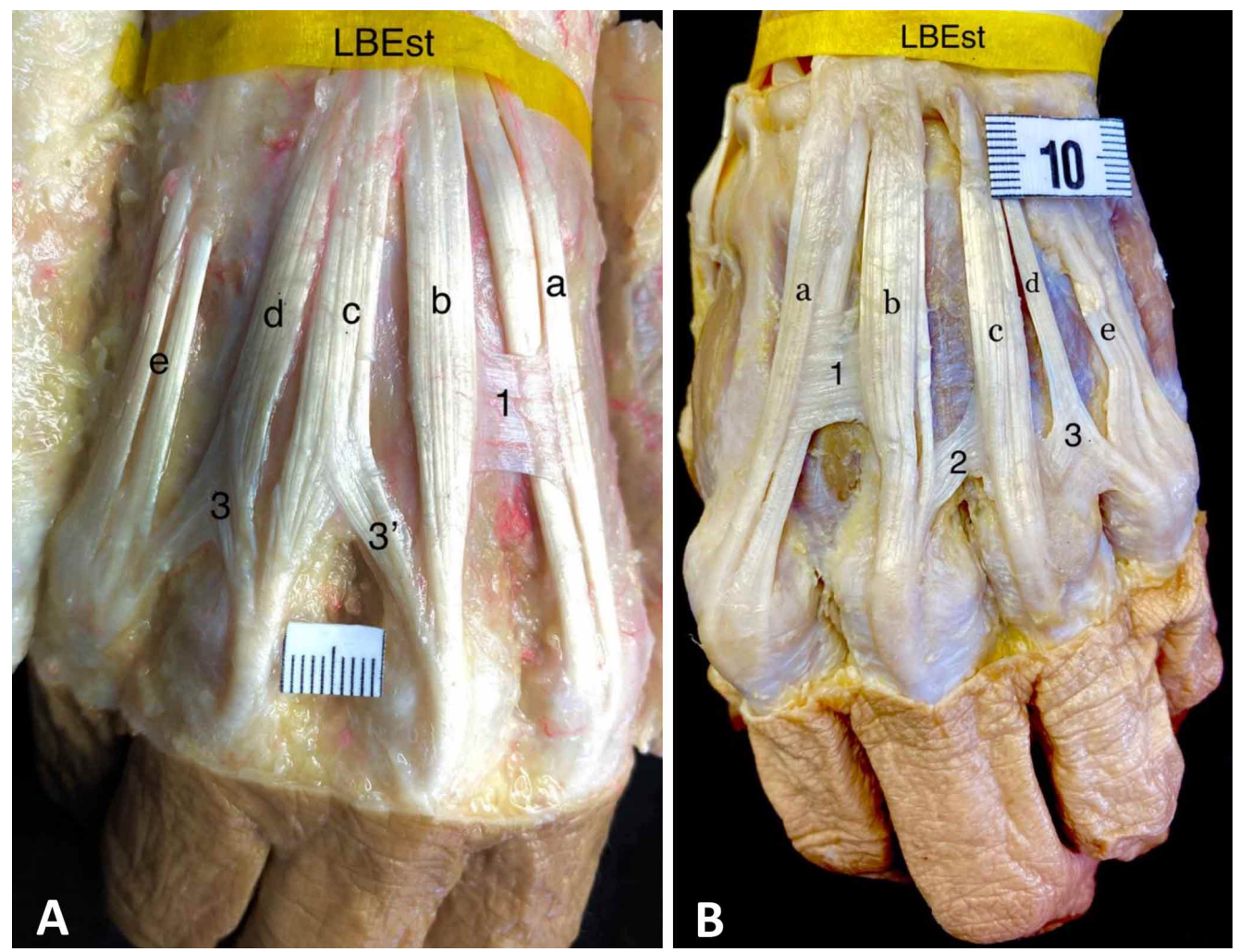

Fig. 3. A. Vista dorsal mano derecha. B. Vista dorsal mano izquierda. a: Tendón del músculo extensor de los dedos (ED) para el dedo índice; b: Tendón del músculo ED para el dedo medio; c: Tendón del músculo ED para el dedo anular; d: Tendón del músculo ED para el dedo mínimo; e: Doble tendón del músculo extensor del dedo mínimo. 1: Conexión intertendinosa (CIT) Tipo 1 en segundo espacio intermetacarpiano (EIMC); 2: CIT Tipo 2 en tercer EIMC; CIT Tipo 3 “y” en tercer EIMC; 3': CIT Tipo 3 " $r$ ” en cuarto EIMC. LBEst: Línea biestiloidea. 


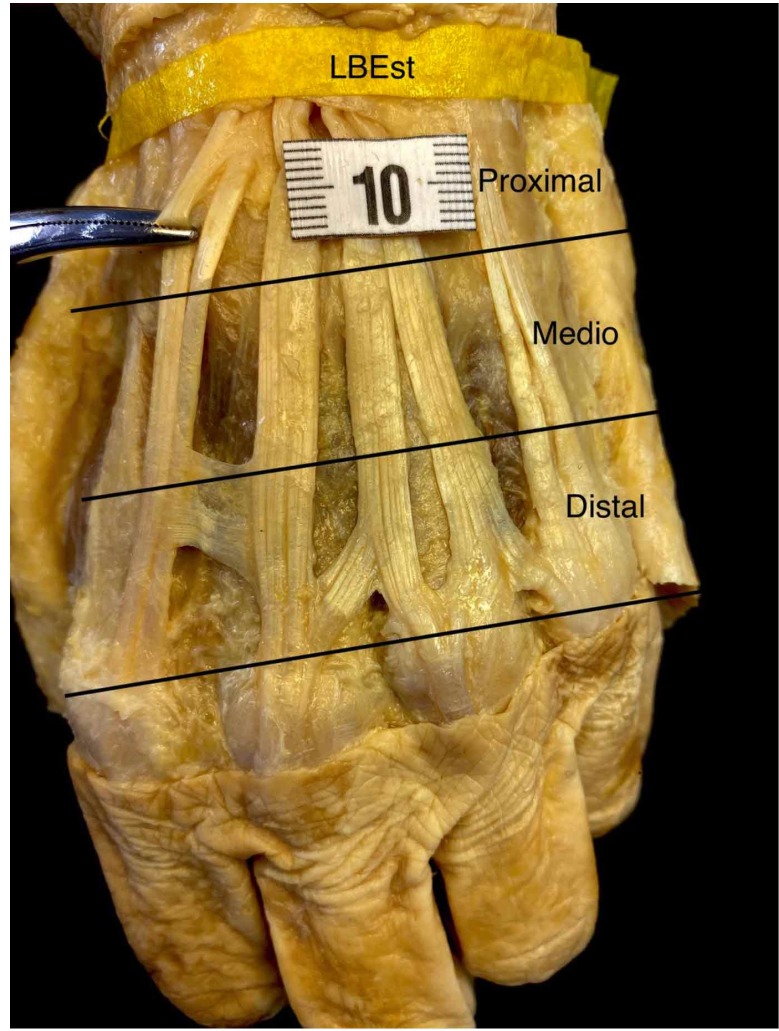

Fig. 4. Vista dorsal mano izquierda. Se detallan la división del dorso de la mano en tercios: proximal, medio, distal. LBEst: Línea biestiloidea.

manos, mientras que el tipo 2 alcanzó $67 \%$. En esta misma línea, la forma"r" del tipo 3 se encontró en un $17 \%$ versus la forma "y" que se halló en el $8 \%$ de los casos. Esta distribución morfológica en el cuarto EIMC fue de 9 $\%$ para el tipo $1,22 \%$ para el tipo $2,43 \%$ para el forma "r" del tipo 3 y $26 \%$ para la forma "y".

Los datos biométricos de las longitudes lateral y medial, promedio de la longitud total y el ancho de las CIT que se localizaron en el segundo, tercer y cuarto EIMT se encuentran resumidas en la Tabla I.

En relación a los EIMC y considerando a la articulación MCF y la LBEst como puntos de referencia, las distancias promedio entre éstas y las CIT se expresan en la Tabla II.

Además, se localizó la distribución de CIT según los tercios del dorso de la mano (Fig. 4). Al respecto, en el segundo EIMC el $18 \%$ de las CIT se localizaron en el tercio distal mientras que el $82 \%$ restante lo hizo en el tercio medio. En el caso del tercer EIMC, el $96 \%$ de las CIT se localizaron en el tercio distal y el $4 \%$ restante se presentó en el tercio medio. Esta distribución de localización se repitió en el cuarto EIMC. Cabe destacar que ninguna CIT se localizó en el tercio proximal del dorso de la mano. Considerando la clasificación según forma se determinó que el 37 $\%$ de las CIT transversas se encontraron en el tercio distal, mientras que el $63 \%$ se ubicaron en el tercio medio. Para el caso de las CIT oblicuas, el $93 \%$ se localizó en el tercio distal y el $7 \%$ restante lo hizo en el tercio medio. Este mismo análisis se realizó según la distribución de forma de von Schroeder et al., encontrándose que el tipo 1 se localizó en un $70 \%$ en el tercio medio y un $30 \%$ en el tercio distal, mientras que el tipo 2 se localizó en un $15 \%$ en el tercio medio y un $85 \%$ en el distal. En relación al tipo 3 "r" este se ubico en un $100 \%$ en el tercio distal, y el tipo 3 "y" en un $12 \%$ en el tercio medio y $88 \%$ de los casos, en el tercio distal.

En relación a las variantes anatómicas, encontramos que en el $42 \%$ de las manos, el músculo ED no presentó tendón para el dedo mínimo. Por otra parte en un $13 \%$ de las manos se presentaron tendones supernumerarios para el dedo medio. En dos de ellos este tendón provenía desde un vientre que se desprendió del músculo propio del dedo índice. En el caso restante el tendón supernumerario provenía de un músculo extensor corto del dedo medio. No se presentaron CIT entre los tendones del músculo ED estos tendones supernumerarios.

Tabla I. Promedio de medidas biométricas de las conexiones intertendinosas en cada espacio intermetacarpiano (EIMC).

\begin{tabular}{lcccccccc}
\hline EIMC & $\begin{array}{c}\text { Ancho } \\
(\mathrm{mm})\end{array}$ & D.E & $\begin{array}{c}\text { Longitud } \\
(\mathrm{mm})\end{array}$ & D.E & $\begin{array}{c}\text { Longitud lateral } \\
(\mathrm{mm})\end{array}$ & D.E & $\begin{array}{c}\text { Longitud medial } \\
(\mathrm{mm})\end{array}$ & D.E \\
\hline Segundo & 8,16 & 1,94 & 13,71 & 4,70 & 15,33 & 4,93 & 15,48 \\
Tercero & 10,94 & 4,39 & 6,29 & 3,26 & 8,59 & 3,19 & 10,27 \\
Cuarto & 7,00 & 3,06 & 7,08 & 4,42 & 7,30 & 4,55 & 10,23 & 4,25 \\
\hline
\end{tabular}

Tabla II. Promedio distancias de las conexiones intertendinosas hacia articulación metacarpofalángicas (MCF) y a la línea biestiloidea (LBEst).

\begin{tabular}{|c|c|c|c|c|c|c|c|c|}
\hline EIMC & $\begin{array}{c}\text { Distancia } \\
\text { lateral MCF } \\
(\mathrm{mm})\end{array}$ & D.E & $\begin{array}{c}\text { Distancia Medial } \\
\text { MCF } \\
(\mathrm{mm})\end{array}$ & D.E & $\begin{array}{c}\text { Distancia lateral } \\
\text { LBEst } \\
(\mathrm{mm})\end{array}$ & D.E & $\begin{array}{c}\text { Distancia Medial } \\
\text { LBEst } \\
(\mathrm{mm})\end{array}$ & D.E \\
\hline Segundo & 29,52 & 4,95 & 29,36 & 5,11 & 46,18 & 8,85 & 45,48 & 8,48 \\
\hline Tercero & 18,09 & 5,09 & 15,81 & 6,37 & 51,62 & 6,57 & 56,34 & 8,28 \\
\hline Cuarto & 8,40 & 6,97 & 13,68 & 3,92 & 53,53 & 6,34 & 54,82 & 6,27 \\
\hline
\end{tabular}




\section{DISCUSIÓN}

La obtención de la extensión independiente de los dedos ha sido motivo de debate desde hace varias décadas. En el siglo XIX diferentes médicos realizaron cirugías para mejorar la libertad de movimiento de los dedos, en particular, de pianistas que deseaban la independencia de la extensión del dedo anular, sin embargo, dicha intervención fue ampliamente cuestionada por su escaso éxito. En este sentido estudios como el de von Schroeder \& Botte (1993), en donde se analizó el comportamiento de los dedos sometidos a una tracción de los vientres musculares del músculo ED de muestras cadavéricas; concluyeron que la interacción funcional entre dedos adyacentes se debe a la existencia de CIT. En este sentido reportaron que la transmisión de esta tracción disminuyó cuando se seccionaron dichas conexiones. A lo anterior agregaron que la menor independencia del dedo anular es producto de que las CIT son más gruesas hacia medial, lo que se debe básicamente a su asociación con las maniobras de agarre por sobre las actividades de pinza digital. Por su parte Keen \& Fuglevand (2003), en un estudio de estimulación eléctrica del mismo músculo en cuatro voluntarios sanos, establecieron que la influencia de las CIT en la redistribución de fuerzas es menor, siendo los factores neuronales los principales responsables de la independencia de la extensión de un dedo respecto los demás. A pesar de ello, existe un consenso respecto del rol biomecánico de las CIT en la estabilización de la articulación MCF, evitando la subluxación de los tendones extensores, lo que colabora en la correcta ejecución de la extensión de los dedos (Chinchalkar et al.).

Por otra parte, diversas han sido las clasificaciones que buscan agrupar a las CIT según su forma. En este sentido Wehbé (1992), estableció que además de describir a las CIT según su disposición transversa u oblicua, éstas se diferencian según su aspecto y grosor. En esta línea, propuso la existencia de tres categorías: a) fascia, b) ligamento y c) tendón. Lo anterior reflejó la existencia de diferencias de grosor o de su conformación histológica. A pesar de disponer de esta clasificación, los investigadores del área han utilizado ampliamente lo propuesto por von Schroeder et al., quienes clasificaron morfologicamente las CIT en tipo 1, 2, 3 "r" y 3 "y" (Hirai et al.; Celik et al.; Pinar et al.; Palatty et al.; Kalkisim et al). Consideramos que dicha clasificación es adecuada no solo porque permite diferenciar la forma, sino que también describe la disposición de la CIT.

Tomando esta clasificación, nuestra investigación en 24 manos, registró la presencia de 69 CIT, de las cuales un $29 \%$ correspondió al tipo 1, 39 \% al tipo 2, $20 \%$ a la forma "r" del tipo 3 y $12 \%$ de la forma "y" del mismo tipo. Dicha distribución no pudo ser contrastada ya que lo diversos estudios describen la distribución de CIT respecto del EIMC en la que se disponen. En este sentido, si analizamos las CIT del segundo EIMC, nuestro reporte coincide con von Schroeder et al., Hirai et al., Celik et al., Pinar et al., Palatty et al. y Kalkısim et al., en que la CIT tipo 1 es la que predomina en ese espacio, alcanzando en nuestro reporte un 66,7 $\%$ de los casos, cifra que se asemeja a los informado por Hirai et al. y Palatty et al. Al analizar y comparar los resultados obtenidos en el tercer EIMC, si bien coincidimos en que la CIT más frecuente fue la tipo 2, nuestra cifra de 66,7 $\%$ es diferente a los restantes estudios, los cuales presentaron un intervalo de frecuencia entre 40 y $59 \%$. De este grupo de autores, los hallazgos de Hirai et al., reportaron que el tipo de CIT más frecuente fue la forma " $r$ " del tipo 3 con un $68 \%$ de los casos.

Lo anterior permite inferir que existe posibilidad de encontrar variaciones anatómicas respecto de la morfología de las CIT, lo cual se puede traducir en diferencias funcionales. Para el caso del cuarto EIMC, nuestro estudio coincide con von Schroeder et al. y Palatty et al., respecto de que la forma predominante fue la 3 " $\mathrm{r}$ "; versus lo descrito por Celik et al., Hirai et al. y Pinar et al., respecto de que la forma predominante fue la forma " $y$ " del tipo 3. Otro aspecto que refuerza la opción de encontrar diferencias anatómicas se asocia al porcentaje de ausencia de las CIT en el segundo, tercer y cuarto EIMC. Al respecto, nuestra serie encontró que las CIT se encuentran en más de un $90 \%$ de los EIMC mencionados, cifra muy alejada del $38,8 \%$ de ausencia reportado por Celik et al, en el segundo EIMC y por sobre todo por el 43,8\% de ausencia reportado por Hirai et al., en el cuarto EIMC (Tabla III).

En este mismo sentido, se evidencia un consenso respecto de la ausencia de estas CIT en el primer EIMC, descripción que difiere con Steichen \& Petersen, quienes reportaron la existencia de una CIT en este espacio, conectando los tendones del músculo extensor largo del pulgar y el tendón del músculo ED dirigido al dedo índice. Dicha descripción no fue observada en nuestra investigación.

Este análisis de ubicación de las CIT en los diferentes EIMC refuerza lo planteado por von Schroeder \& Botte (2001) respecto de que el dedo anular es el menos independiente producto de las CIT que presenta. Lo anterior se fundamenta en el hecho de que la disposición de las CIT tipo 2 y tipo 3 que tiene este tendón con sus tendones adyacentes es opuesta a la dirección de tracción del tendón del músculo EC para este dedo. Lo anterior generaría una inhibición de la extensión del dedo anular cuando los dedos adyacentes están flexionados. En este sentido, la situación del dedo índice es contraria, ya que su mayor movilidad se debería por 
una parte a que recibe un tendón adicional desde el músculo extensor propio del índice y en segundo lugar por la forma transversa de las CIT que unen el tendón del ED de este dedo con el que está destinado al dedo medio de la mano.

En relación a los aspectos biométricos de longitud total y ancho de las CIT existen importantes diferencias entre nuestros hallazgos con los reportados por Von Schroeder et al., Celik et al., Pinar et al. y Palatty et al. En este sentido en nuestra serie, las CIT del segundo EIMC, que en su mayoría fueron tipo 1, presentaron un promedio de longitud total de $13,43 \mathrm{~mm}$ y un ancho de $7,77 \mathrm{~mm}$, en cambio, los autores mencionados cifraron estas mediciones entre 6,4 y $8,7 \mathrm{~mm}$ para la longitud total y un intervalo que fluctuó entre 9 y $12 \mathrm{~mm}$ para el ancho. Para las CIT tipo 2 que predominaron en el tercer EIMC, las cifras fueron similares, ya que en nuestra investigación la longitud alcanzó un promedio de 7,72 $\mathrm{mm}$ y el ancho promedio $8,93 \mathrm{~mm}$, mientras que la cifras del grupos de autores se presentó entre 8 y $11 \mathrm{~mm}$ para la longitud total y entre 5 y $8,7 \mathrm{~mm}$ su el ancho de la CIT. En el caso de las CIT del cuarto EIMC se evidenciaron diferencias. La longitud total de las CIT tipo 3 "r" de nuestro estudio se presentó en promedio a 5,1 mm y el ancho alcanzó 9,19 $\mathrm{mm}$; en cambio, la longitud total reportada por los autores antes mencionados es cercana a los $12 \mathrm{~mm}$, mientras que el ancho varió entre 3,8 y 4,3 $\mathrm{mm}$ para la forma 3 " $r$ ". Misma situación se presentó en la forma "y" del tipo 3. Mientras que en nuestro estudio la longitud total promedio fue de $6,78 \mathrm{~mm}$ y el ancho alcanzó 9,75 , $\mathrm{mm}$, lo reportado en los mencionados estudios fue entre 10 y $15 \mathrm{~mm}$ para la longitud y entre 3,6 y 4,2 mm para el ancho de la CIT. Al respecto consideramos que las diferencias entre las mediciones de los estudios de Von Schroeder et al., Celik et al., Pinar et al. y Palatty et al. se obtuvieron siguiendo una metodología que difiere a lo informado en nuestro estudio. En este sentido nuestra metodología se adhiere a los reportado por Kalkısım et al., quienes establecieron que la longitud se obtiene entre el punto medio de las inserciones lateral y medial de cada CIT, indistinto cómo se orienten las fibras de la misma. Lo anterior determina que la longitud no se asocia necesariamente al eje de la estructura, sino que debe ser medida en sentido proximal distal. Asimismo, el ancho de la CIT debe considerar un eje medial lateral. Consideramos importante establecer una metodología común que permita establecer comparaciones bajo los mismos parámetros.

Respecto de la presencia de variantes anatómicas, en nuestro estudio identificamos que un $13 \%$ de los casos presentó tendones supernumerarios, los cuales no incluyeron la existencia de CIT con tendones adyacentes. En la misma línea, en un $42 \%$ de nuestros casos, el músculo ED no presentó tendón para el dedo mínimo, cifra inferior al $61 \%$ y 71 \% reportados por von Schroeder \& Botte (2001) y Gövsa et al. (2011), respectivamente. Adicional a ello, von Schroeder \& Botte (1995) reportaron que en el $96 \%$ de sus casos, esta ausencia se vinculó con la existencia de una CIT tipo 3 "r", la cual se dirigió desde el tendón que el músculo ED presenta al dedo anular para finalizar en el dedo mínimo. Este tipo de CIT existió en la totalidad de las manos que no presentaron tendón desde el músculo ED hacia el dedo meñique.

Respecto de las distancias entre las CIT y los puntos de referencia coincidimos con lo reportado por von Schroeder et al., quienes utilizando la articulación radiocarpiana como referencia establecieron que las CIT del segundo, tercer y cuarto EIMC se ubicaron a 55, 60 y $61 \mathrm{~mm}$, respectivamente. En este sentido nuestros hallazgos al respecto fueron levemente inferiores, lo que puede estar asociado a que nuestra referencia fue la LBEst, la cual queda dispuesta levemente distal a la línea articular de la articulación radiocarpiana.

Es este mismo sentido, es importante destacar que nuestro estudio describe la localización de las CIT según los tercios del dorso de la mano. Para el caso del segundo 
EIMC el $82 \%$ de las CIT se localizó en el tercio medio, mientras que el $96 \%$ de las CIT del tercer y cuarto EIMC se localizaron en el tercio distal, no encontrando CIT en el tercio proximal de dichos EIMC. Al respecto no encontramos reportes que informaran de la localización de estas CIT siguiendo esta metodología.

Finalmente, consideramos que las descripciones de las CIT resultan de interés para la planificación de las cirugías del dorso de la mano, principalmente aquellas que involucren tenolisis o la realización de transferencias tendinosas. Asimismo, es importante que futuros estudios se enfoquen en los aspectos histológicos de estas CIT, lo cual permitirá evaluar el uso de estas estructuras en procedimientos reconstructivos.

BRUNA, A.; RIVEROS, A. \& OLAVE, E. Biometry of the intertendinous connection of the dorsum of the human hand. Int. J. Morphol., 38(5):1165-1172, 2020.

SUMMARY: Intertendinous connections (IC) are narrow connective tissue bands extending between the extensor digitorum (ED) tendons. These bands are located superficially on the dorsum of the second, third and fourth intermetacarpal (IMC) space, presenting important functions such as collaborating in the stabilization of the metacarpophalangeal joint (MCP) and allowing the redistribution of extensor forces on this joint. The objective of the present study was to establish the biometric characteristics of IC. To do this, 24 formalized upper limbs are used, belonging to the Anatomy laboratory of the Universidad Andrés Bello, Viña del Mar, Chile. Lateral and medial insertion lengths, width and total length of each IC were recorded. Following two criteria, IC were classified according to morphology and were also located according to thirds of the back of the hand. 69 IC were found, 39 $\%$ presented a transverse arrangement and $61 \%$ an oblique arrangement. In relation to the morphological classification of IC, $29 \%$ of type 1 were identified, $39 \%$ of type 2 . In the case of type 3, $20 \%$ presented the form "r" and $12 \%$ the form " $y$ ". Regarding its location, it was established that in the second IMC space, $18 \%$ of the IC were located in the distal third of the back and $82 \%$ in the middle third. In the third IMC space, $96 \%$ of the IC was located in the distal third and $4 \%$ in the middle third. In the fourth IMC space, $96 \%$ were located in the distal third and 4 $\%$ in the middle third. Considering the biestiloid line as a reference, the IC in the second IMC space considers an average width of $8.16+1.94 \mathrm{~mm}$ and an average total length of $13.71+4.70 \mathrm{~mm}$. In the third IMC space, an average of $10.94+4.39 \mathrm{~mm}$ for the width and $6.29+3.26 \mathrm{~mm}$ of average total length. In the fourth IMC space the average total width of $7.00+3.06 \mathrm{~mm}$ and the average total length of $7.08+4.42 \mathrm{~mm}$. These findings provide data regarding the biometrics and location of IC, which is useful in surgical repair procedures on the back of the human hand.

KEY WORDS: Anatomy; Intertendinous connections; Juncturae tendinum; Extensor digitorum muscle; Biometry.

\section{REFERENCIAS BIBLIOGRÁFICAS}

Celik, S; Bilge, O; Pinar, Y \& Govsa, F. The anatomical variations of the extensor tendons to the dorsum of the hand. Clin. Anat., 21(7):652-9, 2008.

Chinchalkar, S. J.; Barker, C. A. \& Owsley, B. Relationship between juncturae tendinum and sagittal bands. J. Hand Microsurg., 7(1):96-101, 2015.

Comité Federal sobre Terminología Anatómica. Terminologia Anatomica. Buenos Aires, Médica Panamericana, 2001.

Godwin, Y. \& Ellis, H. Distribution of the extensor tendons on the dorsum of the hand. Clin. Anat., 5(5):394-403, 1992.

Gövsa, F.; Pinar, Y.; Çelik, S.; Bilge, O. \& Sezak, M.Anatomical similarity between tendons and Type 3 intertendinous connections: suitability as local donor tissue. Acta Orthop. Traumatol. Turc., 45(5):370-5, 2011.

Hirai, Y.; Yoshida, K.; Yamanaka, K.; Inoue, A.; Yamaki, K. \& Yoshizuka, M. An anatomic study of the extensor tendons of the human hand. J. Hand Surg. Am., 26(6):1009-15, 2001.

His, W. Nomina Anatomica. Leipzig, Verlag von Veit \& Comp., 1895.

Kalkısım, S. N.; Çan, M. A.; Uzun, Ö. \& Öksüz, C. E. Morphological and morphometric evaluation of intertendinous connections among extensor tendons in fetal hands. Surg. Radiol. Anat., 40(9):979-88, 2018.

Keen, D. A. \& Fuglevand, A. J. Role of intertendinous connections in distribution of force in the human extensor digitorum muscle. Muscle Nerve, 28(5):61422, 2003.

Le Double, A. F. Traité des Variations Système Musculaire de l'Home et de Leur Signification au Point de Vue de LlAnthropologie Zoologique. Paris, Schleicher frères, 1897.

Llusa, M.; Merí, À. \& Ruano, D. Manual y Atlas Fotográfico de Anatomía del Aparato Locomotor. Madrid, Médica Panamericana, 2004.

Palatty, B. U.; Raveendranath, V. \& Manjunath, K. Y. A morphological study of juncturae tendinae of hand in adults and fetuses. People J. Sci. Res., 7(1):126, 2014

Pinar, Y.; Bilge, O.; Govsa, F.; Celik, S. \& Aktug, H. Anatomo-histological analysis of the juncturae and their relations to the extensor tendons to the dorsum of the hand. Surg. Radiol. Anat., 31(2):77-83, 2009.

Rouvière, H. \& Delmas, A. Anatomía Humana: Descriptiva, Topográfica y Funcional. Vol. 3. $11^{\mathrm{a}}$ ed. Barcelona, Masson, 2005.

Standring, S. Gray's Anatomy. The Anatomical Basis of the Clinical Practice. $40^{\mathrm{a}}$ ed. Edinburgo, Elsevier Churchill Livingstone, 2016.

Steichen, J. B. \& Petersen, D. P. Junctura tendinum between extensor digitorum communis and extensor pollicis longus. J. Hand Surg. Am., 9(5):674-6, 1984.

Testut, L. \& Latarjet, A. Tratado de Anatomía Humana. Vol. 3. 9ª ed. Barcelona, Salvat, 1972.

Tountas, C. \& Bergmann, R. Anatomic Variations of the Upper Extremity. Londres, Churchill Livingstone, 1993.

von Schroeder, H. P. \& Botte, M. J. Anatomy and functional significance of the long extensors to the fingers and thumb. Clin. Orthop. Relat. Res., (383):74$83,2001$.

von Schroeder, H. P. \& Botte, M. J. Anatomy of the extensor tendons of the fingers: variations and multiplicity. J. Hand Surg. Am., 20(1):27-34, 1995.

von Schroeder, H. P. \& Botte, M. J. The functional significance of the long extensors and juncturae tendinum in finger extension. J. Hand Surg. Am., 18(4):641-7, 1993.

von Schroeder, H. P.; Botte, M. J. \& Gellman, H. Anatomy of the juncturae tendinum of the hand. J. Hand Surg. Am., 15(4):595-602, 1990.

Wehbé, M. Junctura anatomy. J. Hand Surg. Am., 17(6):1124-9, 1992.

Yammine, K. The anatomy and prevalence of the juncturae tendinum in the hands A systematic review and meta-analysis. Eur. J. Anat., 19(4):351-9, 2015.

Zancolli, E. Anatomía Quirúrgica de la Mano. Atlas Ilustrado. Buenos Aires, Médica Panamericana, 2014.

Dirección para correspondencia:

Dr. Enrique Olave

Facultad de Medicina

Universidad de La Frontera

Temuco - CHILE

Recibido : 11-04-2020

Aceptado: 10-05-2020

Email: enrique.olave@ufrontera.cl 